\title{
INTEGRAÇÃO DAS DISCIPLINAS DA ÁREA DE RECURSOS HÍDRICOS NO PROCESSO DE FORMAÇÃO DO ENGENHEIRO CIVIL EM UM CURRÍCULO CONSTRUÍDO POR COMPETÊNCIAS
}

Mariane Kempka -marianekempka@utfpr.edu.br*

Universidade Tecnológica Federal do Paraná, Coordenação de Engenharia Civil* Avenida Professora Laura Pacheco Bastos, 800.* 85053-525 - Guarapuava-Paraná*

Jóice Cristini Kuritza - joicekuritza@utfpr.edu.br* Universidade Tecnológica Federal do Paraná, Coordenação de Engenharia Civil* Avenida Professora Laura Pacheco Bastos, 800. * 85053-525 - Guarapuava-Paraná*

Viviane Chulek - vivianechulek@utfpr.edu.br* Universidade Tecnológica Federal do Paraná, Coordenação de Engenharia Civil* Avenida Professora Laura Pacheco Bastos, 800. * 85053-525 - Guarapuava-Paraná*

Marcela Maier Farias Czap - mfarias@utfpr.edu.br* Universidade Tecnológica Federal do Paraná, Coordenação de Engenharia Civil* Avenida Professora Laura Pacheco Bastos, 800. * 85053-525 - Guarapuava-Paraná*

Resumo: Os cursos de engenharia no Brasil devem seguir as Diretrizes Curriculares Nacionais $(D C N)$ instituídas pelo Conselho Nacional de Educação (CNE) por meio da Resolução $n^{\circ} 2$, de 2019. Após a publicação desta resolução, as Instituições de Ensino Superior precisaram encontrar estratégias de capacitação dos docentes para a nova proposta curricular, ampliada para habilidades e competências, que incluam além do conhecimento (saber), também o saberfazer e o saber-ser. Especialmente, para o curso de Engenharia Civil, da Universidade Tecnológica Federal do Paraná (UTFPR), campus Guarapuava, a reformulação do Projeto Pedagógico de Curso (PPC) passou pela consolidação do perfil do egresso, competências e elementos de competência do curso. Neste trabalho, apresenta-se a reestruturação das disciplinas da área de Recursos Hídricos com os respectivos período do curso e carga horária, ainda, expõe-se os elementos de competência associados às disciplinas. São exibidos, também, os conteúdos da disciplina de Mecânica dos Fluidos, escritos na forma de temas de estudo, e os resultados de aprendizagem, os quais possibilitam ao estudante compreender o que será discutido e quais habilidades deverão ser desenvolvidas no decorrer da disciplina.

Palavras-chave: Projeto pedagógico de curso. Competências. Recursos hídricos.

\section{INTRODUÇÃO}

A publicação das novas Diretrizes Curriculares pelo MEC, Resolução no 2, de 24 de abril de 2019, impôs aos Colegiados e aos Núcleos Docentes Estruturantes (NDE) a reformulação dos Projetos Pedagógicos de Cursos (PPC) vigentes com mudança na concepção da formação 
de engenheiros nas instituições de ensino superior (IES). A nova diretriz exige que os cursos de engenharia devem contemplar em seus PPCs as atividades de aprendizagem que assegurem o desenvolvimento das competências estabelecidas no perfil do egresso. Na Universidade Tecnológica Federal do Paraná (UTFPR), o Projeto Pedagógico Institucional expõe que o conceito de competência não se limita ao saber fazer, mas envolve também as atitudes de responsabilidade social, ética do comportamento, convivência participativa, cuidado com o meio ambiente, entre outros (UTFPR, 2019).

Alcançar competências profissionais, nesse sentido, pressupõe muito mais que a apropriação de um conhecimento científico. Implica a necessidade de que o estudante mobilize e integre esses conhecimentos em situações de uso realistas situadas em um contexto social, cultural, econômico e científico que permeiam a vida profissional e portanto também devem permear o processo de formação. Na Universidade Tecnológica Federal do Paraná, campus Guarapuava, o colegiado, o NDE e os professores das áreas específicas da engenharia civil atuaram coletivamente na reestruturação do PPC vigente, com vistas à inovação curricular, à redução da taxa de evasão e ainda o direcionamento para as demandas do mercado de trabalho.

A reestruturação do PPC seguiu o modelo apresentado em três etapas de uma Oficina de Design de Curso promovida pelo Departamento de Educação (DEPEDUC) da Pró-reitoria de Graduação (PROGRAD) da UTFPR, na qual participaram alguns professores do NDE. Na ocasião, foram construídos o Perfil do Egresso, as Competências e os Elementos de Competência, fundamentados no Plano Pedagógico Institucional (PPI), nas Diretrizes Curriculares Nacionais (DCNs) e no contexto de atuação do engenheiro civil no Brasil.

Neste artigo são apresentadas as Competências e os Elementos de Competência (EC) aos quais as disciplinas da área de Recursos Hídricos estão ligadas. Também são apresentados os Temas de Estudo (TE) e os Resultados de Aprendizagem (RA) que foram elaborados na reestruturação destas disciplinas.

\section{A CONSTRUÇÃO DO CURRÍCULO POR COMPETÊNCIAS}

A organização curricular, segundo Lopes (2008), é baseada na lógica das ciências ou na natureza do conhecimento, enquanto currículos integrados são baseados nos interesses e necessidades dos estudantes, além de considerar a relevância social do conhecimento.

Considerando o propósito da educação superior na qual a vinculação entre trabalho e educação definem o programa formativo sistemático que proporciona o desenvolvimento do estudante, a construção do currículo por competências pode ser estruturada por diferentes perspectivas teóricas, mas a integração ocorre na valorização das experiências dos estudantes.

Durand (1999) divide o conceito de competência em três subconceitos: o conhecimento (saber), o saber-fazer e atitude (saber ser). O saber relaciona-se aos conhecimentos que são historicamente legitimados como objeto de estudo e que já fazem parte dos currículos acadêmicos. O saber-fazer refere-se a um agir, seja de forma motora ou cognitiva, pautado na mobilização, integração e transferência de conhecimentos para diferentes situações. A integralização da competência se alcança com o saber-ser entendida como as caraterísticas dos estudantes que podem ser objetivos da formação a serem construídos ao longo do processo, como características pessoais que devem ser atingidos previamente para uma aprendizagem ou ainda como critérios de qualidade particulares a certos desempenhos (SCALLON, 2015).

O cenário do mercado de trabalho atual exige profissionais preparados não só tecnicamente, mas também, capazes de se relacionar eficientemente, que possam trabalhar com equipes multidisciplinares, gerenciar serviços e projetos e identificar o impacto das atividades da engenharia no aspecto socioambiental. Nessa perspectiva, as competências que se deseja 
são, de acordo com o INEP (Instituto Nacional de Estudos e Pesquisas Educacionais Anísio Teixeira), ações e operações utilizadas para estabelecer relações entre objetos, situações, fenômenos e pessoas (BRASIL, 2005), portanto, reestruturar os planos pedagógicos de acordo com as competências desejadas ao egresso, responde às necessidades reais de intervenção da pessoa nos diversos contextos.

Compõem esta organização, também, os elementos de competência (EC) que são estágios a serem desenvolvidos para alcançar a competência. Para isso, os professores estabeleceram um escalonamento dos ECs possibilitando o avanço dos estudantes dentro do processo formativo. A distribuição das disciplinas nas competências e ECs deve ser integrada de acordo com o potencial de contribuição no aperfeiçoamento das competências.

Outro ponto considerado na reestruturação da matriz, diz respeito às atribuições legais do profissional junto aos órgãos e conselhos específicos.

No Paraná, o Conselho Regional de Engenharia e Agronomia do Paraná, no Manual Orientativo de Fiscalização, regulamenta as áreas de atuação e as atividades técnicas pertinentes aos profissionais de Engenharia Civil. $\mathrm{Na}$ área de Recursos Hídricos, destacam-se: aproveitamento, desenvolvimento e preservação de recursos naturais, barragens, delimitação e caracterização de sub-regiões, interpretação hidrológica de bacias fluviais, obras de drenagem urbana, redes de saneamento, reservatórios, cisternas, tratamento de efluentes, entre outras. Em geral, as atividades técnicas englobam projetos estruturais, hidráulicos, elétricos, estudos de viabilidade, drenagem, superficial e profunda, relatórios ambientais (EIA, RIMA), entre outras (CREA-PR, sem data). Portanto, a nova concepção de matriz deve possibilitar a formação de um profissional apto e qualificado a atuar, senão em todas, em grande parte das atribuições a ele conferidas.

\title{
3 METODOLOGIA
}

O trabalho de reestruturação curricular iniciou-se pelo perfil do egresso, construído da seguinte forma:

\begin{abstract}
"Os egressos do Curso de Engenharia Civil são profissionais que se caracterizam por criar soluções de engenharia para o desenvolvimento sustentável do ambiente construído, levando em consideração as demandas da sociedade. Poderão atuar na indústria da construção civil e em órgãos governamentais a ela associados. Para tanto, são capazes de projetar sistemas, produtos e processos relacionados à construção civil considerando a ética, a segurança, a legislação e os impactos ambientais; implementar obras de construção civil de forma responsável, eficaz e com liderança de equipes multiprofissionais; gerenciar organizações correlatas à indústria da construção civil e/ou recursos naturais, de forma empreendedora, inovadora e sustentável".
\end{abstract}

A partir do perfil do egresso foram estabelecidas seis competências que serão desenvolvidas ao longo dos períodos do curso, sendo três delas de formação geral e três de formação específica do engenheiro civil, apresentadas no quadro 1. 
Quadro 1 - Competências esperadas para o egresso de engenharia civil da UTFPR - Guarapuava

\begin{tabular}{|c|c|}
\hline Abreviação & Competência (C) \\
\hline $\begin{array}{l}\text { INVESTIGAR } \\
\qquad(\mathrm{Iv})\end{array}$ & $\begin{array}{l}\text { Investigar problemas de contexto real integrando conhecimentos técnico-científicos da } \\
\text { literatura, selecionando informações relevantes e aplicando em soluções mais } \\
\text { adequadas às contingências com integridade, autonomia reflexiva e senso crítico }\end{array}$ \\
\hline $\begin{array}{l}\text { RESOLVER } \\
\text { (Rv) }\end{array}$ & $\begin{array}{l}\text { Resolver problemas estruturados de diferentes contextos da engenharia, de maneira } \\
\text { autônoma, integrando os fundamentos de química, física e matemática, o raciocínio } \\
\text { lógico e as ferramentas tecnológicas. }\end{array}$ \\
\hline $\begin{array}{l}\text { INTERVIR } \\
\text { (It) }\end{array}$ & $\begin{array}{l}\text { Intervir em situações conflituosas, fundamentado em conhecimentos da dimensão } \\
\text { social, econômica, cultural, tecnológica e ambiental, demonstrando autonomia, } \\
\text { comunicação qualificada, responsabilidade, autoria e autenticidade. }\end{array}$ \\
\hline $\begin{array}{l}\text { EXECUTAR } \\
\text { (Ec) }\end{array}$ & $\begin{array}{l}\text { Executar projetos de construção civil, integrando a interpretação do projeto, o plano } \\
\text { operacional e de viabilidade, a execução, o monitoramento retroalimentado e o } \\
\text { replanejamento, com prioridade na utilização racional de recursos materiais, } \\
\text { financeiros e tecnológicos, e na coordenação regulada de equipes multiprofissionais, } \\
\text { de modo responsável, eficiente e cooperativo. }\end{array}$ \\
\hline $\begin{array}{c}\text { PROJETAR } \\
(\mathrm{Pj})\end{array}$ & $\begin{array}{l}\text { Projetar sistemas em diferentes contextos da construção civil, articulando } \\
\text { adequadamente análise de demanda, diagnóstico de viabilidade, busca de referências, } \\
\text { seleção de concepções e emprego de ferramentas tecnológicas, conforme os aspectos } \\
\text { de funcionalidade, segurança e sustentabilidade, de forma crítico-reflexiva, criativa e } \\
\text { com responsabilidade social. }\end{array}$ \\
\hline $\begin{array}{c}\text { GERENCIAR } \\
(\mathrm{Gr})\end{array}$ & $\begin{array}{l}\text { Gerenciar diferentes organizações correlatas à indústria da construção civil, com visão } \\
\text { sistêmica, empregando planejamento, metodologias, ferramentas gerenciais e } \\
\text { tecnológicas, devidamente validados para tomada de decisão sustentáveis e } \\
\text { inovadoras, com responsabilidade socioambiental, de forma empreendedora, } \\
\text { colaborativa e reflexiva. }\end{array}$ \\
\hline
\end{tabular}

Cada competência foi dividida em elementos de competência (EC) para descrever melhor as etapas de aprendizagem para esta seja alcançada. Ao total, para as seis competências do curso, tem-se 39 elementos de competências os quais estão associados a diferentes disciplinas ao longo do curso. Para cada disciplina, os conteúdos dos planos de ensino foram avaliados no intuito de perceber suas contribuições para as competências desejadas. $\mathrm{O}$ esforço seguinte foi elaborar Temas de Estudo (TE), discutidos em reuniões do NDE para aprimoramento, de forma colaborativa. A partir dos TEs foram produzidos os resultados de aprendizagem (RA), que ligados aos ECs demonstram o potencial de contribuição da disciplina no desenvolvimento das habilidades desejadas no egresso.

\section{RESULTADOS}

A área de Recursos Hídricos e Saneamento Ambiental conta com oito disciplinas, distribuídas ao longo dos 10 períodos, conforme apresentado no quadro 2. 
"Os desafios para formar hoje o engenheiro do amanhã"

Quadro 2 - Disciplinas da área de Recursos Hídricos.

\begin{tabular}{|c|c|c|c|}
\hline Disciplina & Código & Período & Carga Horária (h) \\
\hline Ciências do Ambiente & Camb_3 & $3^{\circ}$ & 30 \\
\hline Mecânica dos Fluidos & Mecf_4 & $4^{\circ}$ & 45 \\
\hline Hidráulica de Condutos Forçados & Hidrco_5 & $5^{\circ}$ & 45 \\
\hline Hidráulica de Canais & Hidrca_6 & $6^{\circ}$ & 45 \\
\hline Hidrologia & Hidrol_7 & $7^{\circ}$ & 60 \\
\hline Saneamento & San_8 & $8^{\circ}$ & 60 \\
\hline Sistemas Hidráulicos Urbanos & Shu_8 & $8^{\circ}$ & 60 \\
\hline Gestão Ambiental & Gamb_9 & $9^{\circ}$ & 30 \\
\hline
\end{tabular}

As oito disciplinas da área contribuirão com o desenvolvimento de dezesseis ECs, distribuídos em todas competências do curso, demonstrando a integração preconizada no processo de construção do currículo. Considerando que, em todo curso, tem-se trinta e nove ECs, as disciplinas abordadas neste trabalho estão vinculadas à formação de $41 \%$ das habilidades e competências dos estudantes. Apesar de contribuir com as seis competências do curso, as disciplinas, em função do caráter e do período em que se inserem, têm maior participação na construção das competências específicas, certificadas no $8^{\circ}$ e $9^{\circ}$ períodos, respectivamente.

No quadro 3 relacionam-se as competências e os elementos de competência às disciplinas da área de recursos hídricos. O vínculo entre o EC e a disciplina pode ser do tipo internalizador ou mobilizador. O tipo internalizador tem processos cognitivos do EC que estão sendo desenvolvidos pela primeira vez, já o mobilizador ocorre quando os recursos adquiridos em disciplinas pregressas são acionados.

Os elementos de competência serão atingidos a partir de Resultados de Aprendizagem (RA), que devem explicitar o desempenho esperado dos estudantes e são parte fundamental para a avaliação da aprendizagem (NICOLA, 2017). Para cada disciplina, a partir dos temas de estudo, os professores da área elaboraram RA's que foram apreciados e aprimorados coletivamente, pelo NDE e pelo Colegiado, para atender às necessidades do curso. 
Quadro 3 - Elementos de competências e disciplinas acionadoras

\begin{tabular}{|c|c|c|c|c|c|c|c|c|c|}
\hline$\cup$ & Elementos de Competência & m & 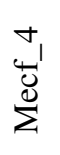 & 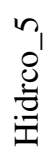 & 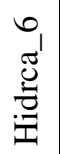 & $\frac{1}{0}$ & $\begin{array}{l}\infty \\
\tilde{E}_{\tilde{n}}\end{array}$ & $\begin{array}{l}\infty \\
\Xi_{1} \\
\Xi^{\prime}\end{array}$ & 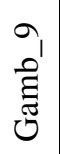 \\
\hline \multirow[b]{2}{*}{$\Xi$} & Problematizar situações de contexto real descritas (Iv_1) & & & & & & & & \\
\hline & $\begin{array}{c}\text { Argumentar, de forma consistente e crítica e pautado em } \\
\text { conhecimentos técnico-científicos da literatura, em defesa de } \\
\text { resultados analisados. (Iv_2) }\end{array}$ & & & & & & & & \\
\hline \multirow[b]{2}{*}{$\vec{\imath}$} & $\begin{array}{c}\text { Interpretar os problemas estruturados no contexto das } \\
\text { Engenharias }\left(\mathrm{Rv}_{-} 1\right)\end{array}$ & & & & & & & & \\
\hline & $\begin{array}{c}\text { Validar resultados relacionando-os ao contexto e à hipótese } \\
\text { selecionada, demonstrando autorregulação durante o } \\
\text { desenvolvimento do processo. }\left(\mathrm{Rv}_{-} 2\right)\end{array}$ & & & & & & & & \\
\hline \multirow[t]{2}{*}{$\Xi$} & $\begin{array}{c}\text { Reconhecer situações de conflito de ordem individual e } \\
\text { coletiva delimitando variáveis em situações de contexto real } \\
\text { (It_1) }\end{array}$ & & & & & & & & \\
\hline & $\begin{array}{c}\text { Definir estratégias de intervenção autênticas e autorais } \\
\text { adequadas à resolução do conflito tratado(It_2) }\end{array}$ & & & & & & & & \\
\hline i & $\begin{array}{l}\text { Monitorar a execução e os resultados alcançados, com base } \\
\text { nos critérios selecionados, replanejando, se necessário. (Ec_1) }\end{array}$ & & & & & & & & \\
\hline \multirow{4}{*}{$\ddot{L}$} & $\begin{array}{c}\text { Analisar as demandas de projetos a partir da identificação das } \\
\text { necessidades e expectativas do público-alvo. }\left(\mathrm{Pj}_{-} 1\right)\end{array}$ & & & & & & & & \\
\hline & $\begin{array}{l}\text { Identificar os parâmetros técnicos, legais e socioeconômicos } \\
\text { necessários para a elaboração do projeto, seus prazos bem } \\
\text { como as ferramentas tecnológicas aplicáveis, com } \\
\text { responsabilidade social durante todas as etapas. }\left(\mathrm{Pj}_{2} 2\right)\end{array}$ & & & & & & & & \\
\hline & $\begin{array}{c}\text { Aplicar modelos espaciais e algébricos que descrevam } \\
\text { adequadamente a funcionalidade dos elementos a serem } \\
\text { projetados. }\left(\mathrm{P} j \__{-} 3\right)\end{array}$ & & & & & & & & \\
\hline & $\begin{array}{c}\text { Dimensionar os elementos dos projetos com ferramentas } \\
\text { tecnológicas adequadas, considerando critérios de segurança e } \\
\text { sustentabilidade, com atitude criativa e crítico-reflexiva. } \\
\left(\mathrm{Pj}_{-} 4\right)\end{array}$ & & & & & & & & \\
\hline \multirow{5}{*}{$\dot{0}$} & $\begin{array}{l}\text { Avaliar o contexto mercadológico da construção civil, } \\
\text { selecionando ferramentas gerenciais e tecnológicas, com foco } \\
\text { na identificação de oportunidades de negócios. }\left(\mathrm{Gr}_{-} 1\right)\end{array}$ & & & & & & & & \\
\hline & $\begin{array}{l}\text { Formular alternativas de solução sustentáveis e inovadoras } \\
\text { para as oportunidades de negócio identificadas, com } \\
\text { responsabilidade socioambiental e de forma empreendedora. } \\
\text { (Gr_2) }\end{array}$ & & & & & & & & \\
\hline & $\begin{array}{c}\text { Monitorar continuamente os indicadores de desempenho, } \\
\text { conforme os critérios do Plano de Ação, reformulando-o com } \\
\text { base nos resultados, de forma reflexiva. }\left(\mathrm{Gr} \_3\right)\end{array}$ & & & & & & & & \\
\hline & $\begin{array}{c}\text { Implementar o Plano de Ação elaborado, de forma } \\
\text { colaborativa. }\left(\mathrm{Gr}_{4} 4\right)\end{array}$ & & & & & & & & \\
\hline & $\begin{array}{l}\text { Documentar o processo de gerenciamento da organização, de } \\
\text { forma reflexiva. }(\mathrm{Gr} \text {-5) }\end{array}$ & & & & & & & & \\
\hline
\end{tabular}


No quadro 4 estão dispostos os temas de estudos e os resultados de aprendizagem da disciplina de Mecânica de Fluidos, com 45 horas, localizada no $4^{\circ}$ período do curso. É válido relatar que, antes da reformulação, a disciplina pertencia ao $5^{\circ}$ período, contudo, a adequação foi necessária para que a disciplina contribuísse, principalmente, com a formação da competência RESOLVER, que mostra-se mais alinhada ao caráter central da disciplina.

Quadro 4 - Temas de estudo e resultados de aprendizagem elaborados para a disciplina de mecânica de fluidos e elementos de competência aos quais contribui

\begin{tabular}{|c|c|c|}
\hline$\bigcup_{\mid I}$ & Temas de Estudo & Resultados de Aprendizagem \\
\hline$\vec{\approx}$ & $\begin{array}{l}\text { TE_1: Definição, grandezas e } \\
\text { propriedades dos fluidos }(5 \\
\text { horas). }\end{array}$ & $\begin{array}{l}\text { RA_1 (TE_1): Identificar a influência das grandezas e das } \\
\text { propriedades dos fluidos considerando as áreas de aplicação } \\
\text { da mecânica dos fluidos no contexto da engenharia civil de } \\
\text { forma crítica. }\end{array}$ \\
\hline $\begin{array}{l}N \\
\vec{\approx}\end{array}$ & $\begin{array}{l}\text { TE_2: Hidrostática no contexto de } \\
\text { estruturas de contenção de água } \\
\text { (10 horas). } \\
\text { TE_4: Conservação da massa e } \\
\text { Análise da energia de escoamento } \\
\text { no contexto do transporte de } \\
\text { fluidos ( } 20 \text { horas). }\end{array}$ & $\begin{array}{l}\text { RA_2 (TE_2): Determinar os esforços em estruturas de } \\
\text { contenção considerando as equações da hidrostática } \\
\text { demonstrando autorregulação. } \\
\text { RA_4 (TE_4): Identificar as condições de aplicação da } \\
\text { análise de energia na resolução de problemas estruturados } \\
\text { no contexto da mecânica dos fluidos com assertividade. } \\
\text { RA_5 (TE_4): Resolver problemas de transporte de fluidos } \\
\text { aplicando conhecimentos da conservação da massa e da } \\
\text { análise de energia demonstrando autorregulação. }\end{array}$ \\
\hline$m$ & $\begin{array}{l}\text { TE_3: Teoria da Semelhança: } \\
\text { análise dimensional e aplicaçôes } \\
\text { (10 horas). }\end{array}$ & $\begin{array}{l}\text { RA_3 (TE_3): Aplicar a teoria da semelhança por meio da } \\
\text { análise dimensional relacionando adequadamente as } \\
\text { grandezas que compõem o escoamento nos problemas da } \\
\text { mecânica dos fluidos com assertividade. }\end{array}$ \\
\hline
\end{tabular}

Uma das inovações dessa nova configuração do PCC é a apresentação de Temas de Estudo, contrário ao tradicional ementário de conteúdos. Os temas de estudo foram concebidos de modo que tenham significado para os estudantes no momento da apresentação do plano de ensino. Além disso, a escrita do TE concilia os conhecimentos à área de atuação do egresso do curso, por exemplo, no TE_2, a Hidrostática será abordada com ênfase ao contexto de estruturas de contenção de água, cujo projeto estrutural e hidráulico é atribuição do engenheiro civil.

A elaboração dos RAs pressupõe que esteja explícito como estes serão mensurados no decorrer da disciplina e seguiu o formato: verbo de operação cognitiva $+\mathrm{TE}+$ qualificador + saber ser. Ponderou-se que os verbos de operação cognitiva devem ser mensuráveis e, também, respeitar a dimensão do processo cognitivo (Taxonomia de Bloom, revisado por Anderson, et al 2001) do elemento de competência ao qual o RA está associado. Assim, verbos cuja avaliação não possa ser observada devem ser evitados, tal como, saber, conhecer, entre outros. Um exemplo é no EC Rv_1 onde propõe-se a interpretação de problemas, cuja dimensão cognitiva do verbo é a compreensão e, portanto, poderiam ser utilizados verbos de dimensão igual ou inferior a esta para o RA. Neste caso, o RA_1 estimula à compreensão por meio da ação de identificar.

O qualificador, por sua vez, deve expressar o contexto, meios, recursos ou grau de exigência do resultado de aprendizagem. Ainda no RA_1, o TE_1 será avaliado considerando 
as áreas de aplicação da mecânica dos fluidos no contexto da engenharia civil. A propriedade Compressibilidade, que trata da variação da massa específica de um fluido, em muitos casos da engenharia civil pode ser desprezada. Por outro lado, na formação de estudantes de outras engenharias, como a aeronáutica, a simplificação desse conceito seria um grande equívoco.

Ao final do RA apresenta-se o saber ser, que está relacionado à atitude a ser desenvolvida pelo estudante durante o processo. Alguns exemplos de saber-ser estão relacionados às atitudes reflexivas, críticas, responsáveis, colaborativas e de autorregulação.

Por fim, a carga horária definida para o TE está atrelada à complexidade do saber. Os temas mais complexos exigem carga horária maior e, por conseguinte, maior número de RAs. Em geral, foram propostos um RA para cada TE, no entanto, para o TE_4, por tratar de um conhecimento mais desafiador, foram propostos dois RAs onde, no primeiro, o estudante identifica as condições de aplicação do TE e, no segundo, resolve os problemas sugeridos.

\section{CONSIDERAÇÕES FINAIS}

As Diretrizes Curriculares Nacionais dos Cursos de Engenharia, publicada em 2019, abrem a perspectiva de um novo entendimento sobre a flexibilidade dos cursos, a importância e a construção coletiva do PPC e a capacitação dos docentes. Elas representam um desafio para as engenharias e, também, uma mudança no modelo de ensino, resultando em uma melhor formação, com maior impacto social da educação em engenharia. Ainda, a abordagem proposta implica em significativa mudança de paradigma, substituindo o enfoque diretamente ligado à aprendizagem tradicional, com ênfase nos conteúdos. As exigências do mercado profissional requerem, além do conhecimento técnico específico, outras competências, entre as quais, o trabalho em equipe, o domínio das novas tecnologias e a concepção de soluções criativas e inovadoras

No processo de reformulação curricular do curso de engenharia civil da UTFPR, campus Guarapuava, as disciplinas da área de Recursos Hídricos, distribuídas ao longo do curso, do $3^{\circ}$ ao $9^{\circ}$ período, estão vinculadas a todas as competências do curso, por meio da ligação com dezesseis elementos de competência ( $41 \%$ do total). Para cada disciplina, a partir da observação dos conhecimentos estruturantes contemplados em seu plano de ensino e dos elementos de competência, foram elaborados e, posteriormente, aprimorados coletivamente, os temas de estudo e os resultados de aprendizagem. Esses elementos indicam as reais possibilidades de implementação curricular, pois relacionam, de forma objetiva, o macro planejamento expresso em forma de Projeto Pedagógico de Curso e o micro planejamento realizado para cada disciplina.

Neste artigo, como forma de exemplificar a metodologia de trabalho, a disciplina de Mecânica de Fluidos teve seus TEs e RAs detalhados. Cabe destacar, no entanto, que todas as disciplinas da área (Quadro 2), passaram pelo processo de reestruturação, observando particularmente os elementos de competência associados, como descrito no quadro 3.

Ainda, a configuração dos resultados de aprendizagem contempla a inserção do saber-ser, demonstrando a intenção pedagógica de formação de um engenheiro civil com visão holística e humanista, crítico, reflexivo, criativo, cooperativo e ético, além da forte formação técnica, conforme preconizado nas DCNs de 2019.

\section{Agradecimentos}

Os autores agradecem ao DEPEDUC/UTFPR pela realização da Oficina de Design de Curso e aos professores do Colegiado e do Núcleo Docente Estruturante do curso de Engenharia 
"Os desafios para formar hoje o engenheiro do amanhã"

Civil da UTFPR-Guarapuava pela dedicação e compromisso ao longo do processo de discussão e reformulação do PPC.

\section{REFERÊNCIAS}

ANDERSON, Lorin W., KRATHWOHL, David R. AIRASIAN, Peter W. A taxonomy for learning, teachingandassessing: a revisonofBloom'sTaxonomyofEducationalObjectives. Nova York: Addison Wesley Longman, 2001. 336 p

BRASIL. Instituto Nacional de Estudos e Pesquisas Educacionais Anísio Teixeira. Exame Nacional do Ensino Médio (Enem): fundamentação teórico-metodológica. Brasília, 2005. Disponível em: <shorturl.at/dtzQ6>, acesso em 23 de junho de 2020.

CONSELHO NACIONAL DE EDUCAÇÃO. Resolução CNE/CES no 02/2019, de 24 de abril de 2019. Institui as Diretrizes Curriculares do Curso de Graduação em Engenharia. Diário Oficial da União, Brasília, 26 de abril de 2019, Seção 1, pp. 43 e 44.

CREA-PR. Conselho Regional de Engenharia e Agronomia do Estado do Paraná. Manual Orientativo de Fiscalização: Câmara especializada de Engenharia Civil. Modalidade Civil. [S.D]. Disponível em: https://www.crea-pr.org.br/ws/wp-content/uploads/2016/12/manualfiscalizacao-CEEC-civil-v2016.pdf. Acessoem: 22 de jun. 2020.

DURAND, T. FormsofIncompetence. In: Sanchez, Ron, andAiméHeene, editors (2000), TheoryDevelopment for Competence-Based Management, Volume 6(A) in Advances in Applied Business Strategy, Lawrence Foster, series editor, Greenwich, CT: JAI Press. Disponível em: 〈shorturl.at/cADFQ〉, acesso em 22 de junho de 2020.

LOPES, A. C. (2008). Políticas de integração curricular. Rio de Janeiro: UERJ.

NICOLA, Rosane de Melo Santo, BEHRENS, Marilda Aparecida. Contribuições da teoria da complexidade para a inovação no planejamento pedagógico do ensino superior. Revista Diálogo Educacional, Curitiba, v. 17, n. 52, p. 357-386, abr./jun. 2017. Disponível em: $\langle$ https://periodicos.pucpr.br/index.php/dialogoeducacional/article/view/9882>, acesso em 22 de junho de 2020.

SCALLON, Gérard. Avaliação da aprendizagem em uma abordagem por competências. Tradução de Juliana Vermelho Martins. Curitiba: PUCPRess, 2015.

UTFPR, Projeto Pedagógico Institucional (PPI) da Universidade Tecnológica Federal do Paraná, Curitiba, 2019.

\section{INTEGRATION OF DISCIPLINES IN THE WATER RESOURCES AREA IN THE CIVIL ENGINEER EDUCATION PROCESS IN A CURRICULUM BUILT BY SKILLS}

Abstract:Engineering courses in Brazil must follow the National Curriculum Guidelines (DCN) instituted by the National Education Council (CNE) through Resolution No. 2, 2019. After the 
publication of this resolution, Higher Education Institutions needed to find strategies for training teachers for the new curricular proposal, expanded to skills and competences, which include not only knowledge (know), but also know-how to do and know-how to be. Especially for the Civil Engineering course, at the Federal Technological University of Paraná (UTFPR), Guarapuava campus, the reformulation of the Pedagogical Course Project (PPC) involved the consolidation of the graduate's profile, competences and competence elements of the course. After that, teachers from different areas restructured their subjects in order to integrate them into the graduate's profile and, thus, enhance the development of students' skills. In this work, the restructuring of the disciplines in the Water Resources area is presented with the respective course period and workload. The elements of competence associated with the disciplines are exposed. Also shown are the contents of the Fluid Mechanics discipline, written in the form of study topics, and the learning results, which enable the student to understand what will be discussed and what skills should be developed during the course.

Keywords:PedagogicalCourse Project. Skills. WaterResources. 